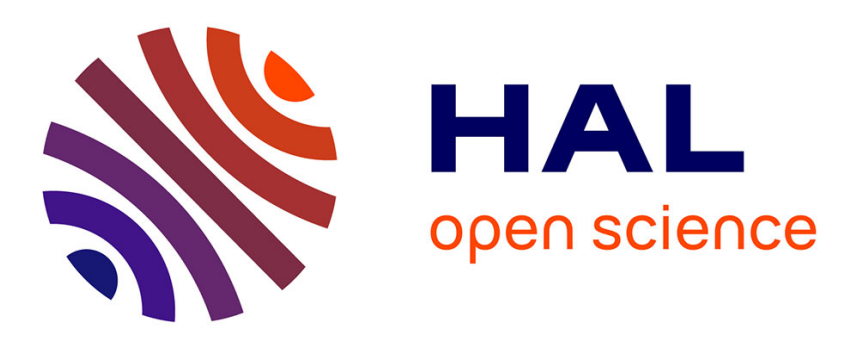

\title{
Filling holes in digitized point cloud using a morphing-based approach to preserve volume characteristics
}

\author{
Yann Quinsat, Claire Lartigue
}

\section{- To cite this version:}

Yann Quinsat, Claire Lartigue. Filling holes in digitized point cloud using a morphing-based approach to preserve volume characteristics. International Journal of Advanced Manufacturing Technology, 2015, 81 (1), pp.411-421. 10.1007/s00170-015-7185-0 . hal-01150322

\section{HAL Id: hal-01150322 \\ https://hal.science/hal-01150322}

Submitted on 11 May 2015

HAL is a multi-disciplinary open access archive for the deposit and dissemination of scientific research documents, whether they are published or not. The documents may come from teaching and research institutions in France or abroad, or from public or private research centers.
L'archive ouverte pluridisciplinaire HAL, est destinée au dépôt et à la diffusion de documents scientifiques de niveau recherche, publiés ou non, émanant des établissements d'enseignement et de recherche français ou étrangers, des laboratoires publics ou privés. 


\title{
Filling holes in digitized point cloud using a morphing-based approach to preserve volume characteristics
}

\author{
Yann Quinsat · Claire lartigue
}

Received: date / Accepted: date

\begin{abstract}
Filling holes is a major challenge to reduce digitizing time and makes the digitized model compatible with applications such as Finite Element (FE) analysis or inertia calculations. Indeed, whatever the sensor used, for accessibility reasons or reflection problems, some parts of the object may be non-measured defining digitizing holes in the digitized point cloud. In this paper a method based on a mesh deformation is proposed to fill the digitized holes. The proposed method relies on the a priori knowledge of the numerical model as a nominal mesh. After identifying the digitized holes and calculating the differences between the nominal mesh and the point cloud, a deformation of the nominal mesh is performed. This deformation is determined by minimizing the energy of deformation of the mesh considered as a lattice. The proposed method is validated on a complex shape. Finally, this method is applied to an industrial part in order to highlight interest for balancing issues.
\end{abstract}

Keywords Digitalization · Energy minimisation · Holes filling $\cdot$ shape preservation

\section{Introduction}

Various types of digitizing systems exist to acquire the shapes of a 3D object. Whatever the sensor used, laser plane, structured light or stereo-vision, optical systems allow the acquisition of a great amount of points representative of the part surfaces in a very short time. Re-

Y. Quinsat

LURPA, ENS Cachan, Université Paris Sud 11

61 avenue du président Wilson, F94235 Cachan, France

Tel.: +33147402213

Fax: +33147402220

E-mail: yann.quinsat@lurpa.ens-cachan.fr searches now focus on using optical measuring systems for in-line production measurements for which measuring time is a critical issue. However, it is commonly admitted that digitized point clouds are noisy, dense and heterogeneous [14], and that for accessibility reasons or reflection problems some parts of the object may be non-measured defining digitizing holes in the point cloud. Holes in the object representation could make further applications, such as Rapid Prototyping [7], Finite Element Analysis (FEA), or volume calculations difficult to achieve [21][26].These holes also contribute to increase the freeform surfaces reconstruction time, which is already a time consuming operation [2]. Numerous studies [14] [6] [13] [18] deal with the definition of automated measuring strategies with the objective of time minimization while increasing the point cloud completeness, but re-digitizing is generally timeconsuming and sometimes incompatible with in-process measurements. Furthermore, some zones could remain inaccessible. Some authors propose to reach the complete 3D object representation by filling the holes [21] [15]. Because of the variety of hole types, a large number of hole-filling algorithms exist, each one more or less dedicated to one specific type[20].

In this study, we use the a priori knowledge of the CAD model of the object under study to overcome the digitizing defects. The algorithm relies on a stress-strain deformation of the nominal geometry performed with the aim of minimizing the differences between the CAD model geometry and the point cloud while preserving the initial topology. It will thus be possible to fill the holes with the deformed geometry. This approach is interesting as it preserves the quality of the identified geometrical characteristics and of the measured dimensions of the object. It is appropriated when the point exploitation requires a complete representation of the 

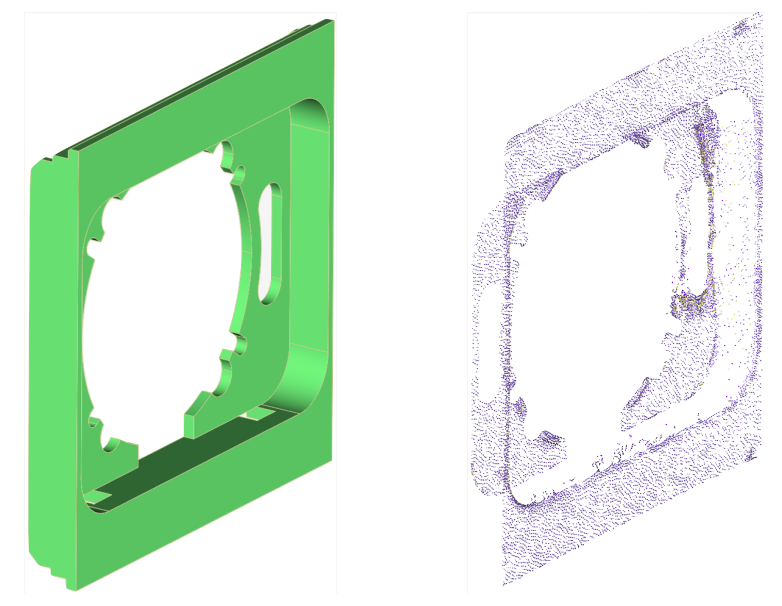

Fig. 1 Digitized holes

object as for FEA, or volume calculation as it is the case in the present paper. In fact, the approach is applied to inertia calculation, first step in crankshaft balancing. As crankshafts are complex parts, some surfaces are difficult to measured, and the digitizing process generally leads to incomplete point cloud presenting digitizing holes. Inertia calculation can only be performed if the discrete data structure is closed. This is performed by filling the holes using the morphing-based approach.

\subsection{Related works}

Methods for hole-filling generally consist of two subproblems: hole detection and reconstruction of the missing regions [19]. The method proposed by Wang and Oliveira, is well-adapted to scanned data. First a mesh is associated to the point cloud. The boundaries of the holes are detected by identifying the boundary edges (edge belonging to only one triangle). A ring of points around the boundary, defining the boundary vicinity, is used to interpolate the missing portion using a Moving Least Squares procedure. Reconstructed patches preserve and smoothly blend with the original model.

Jun [9] bases his approach on the simple principle that a hole can be filled with planar triangulation (such as Delaunay triangulation) if all the boundary edges are projected onto a plane without auto-intersections. As this only works well for simple holes, complex holes are divided into several simple sub-holes that can be filled using the aforementioned method. In a final step, smoothing is applied to refine the model quality. Li et al. [12] propose to first identify feature curves, then use curve blending to complete the missing parts of the feature curves in the holes. A Bézier-Lagrange hybrid patch relying on the feature curve is then constructed to fill the holes. The method preserves the features but is not completely automated. In their approach, Wang et al. [21] define a method well-adapted to restoring missing curves and corners. Missing feature curves (sharp edges)are reconstructed as B-spline curves. Missing corners are found by minimizing the square of the tetrahedron's volume reconstructed from the potential corner and its neighbors. Holes are thus filled using Advancing Front Method. This method is less adapted to complex surfaces. Wang and Hung [20] propose a method based on Grey System Theory to achieve a smooth and continuous hole filling. Grey system theory is used to reduce randomness and increase regularity in the data. A prediction model is used to identify the location of the future element. In their approach, authors consider two prediction models: the normal vector prediction and the angle prediction. Once newly points are added a final smoothing stage is performed but curvature is not controlled. Some authors take advantage of 2D images that have been acquired during the measurement stage to recover the missing zones [15], [17]. The 2D images are used as a set of constraints, and as input of a mesh deformation process which tends to minimize the curvature evolution between the inserted facets and the initial mesh. The mesh deformation relies on a mechanical approach for which the mesh is considered as a bar network. With such an approach, that combines techniques of shape from shading and mesh deformation, the overall shape of the part is preserved. However, it requires the use of two measuring systems at the same time. Some authors use morphing to reconstruct missing data with a specific application in dental surface reconstruction. The objective is to align a scanned tooth to a standard model. The approach begins with a one-to-one mapping of feature points of the standard tooth and the scanned tooth thanks to a Radial basis function to define the relations for all points in both teeth. A global deformation (translation, rotation, and scaling) is performed followed by local adjustments to align the tooth in preparation to the standard one [23]. In this method, the use of morphing permits to fill the holes by taking advantages of the prior knowledge of the general tooth shape. Morphing is more used in computer graphics for mesh deformation. Two main kinds of approaches exist: the geometric methods and the physically-based methods [24]. As far as geometric methods are concerned, lattice or mesh nodes are moved according to geometric constraints to achieve the deformation. In [8], the objective is to relate a template to a target geometry considering some reference points referred to as landmarks. After a general alignment of the landmarks, using translation, rotation and scaling, 
the morphing is performed using the landmarks as constraints. The motion of the mesh nodes is interpolated from the motion of the landmarks based on a Radial Basis function regression to preserve smoothness. The method is applied for human-femur reconstruction. In some cases, the morphing can be restrained to a region of interest while preserving the general shape. Kho and Garland [10] propose an iterative sketch-based deformation. The user draws a reference curve in a zone of interest and a second target curve. The deformation is achieved by deforming the reference curve toward the target one. These methods are generally more suited to visualization and animation.

Physically-based approaches lead to more realistic results as they rely on energy minimization [24], [5], [15], [17]. As for geometric methods, the 3D object is represented as a triangular mesh, but the deformation is made here considering mechanical energy. Most methods only consider the stretching energy which accounts for length changes and add constraints to preserve the curvature evolution. In our approach, all types of mechanical energy are taken into account: stretching, bending and torsion energy. Stretching corresponds to length changes, while bending and torsion ensure the overall shape preservation and continuity in curvature evolution.

\subsection{Method overview}

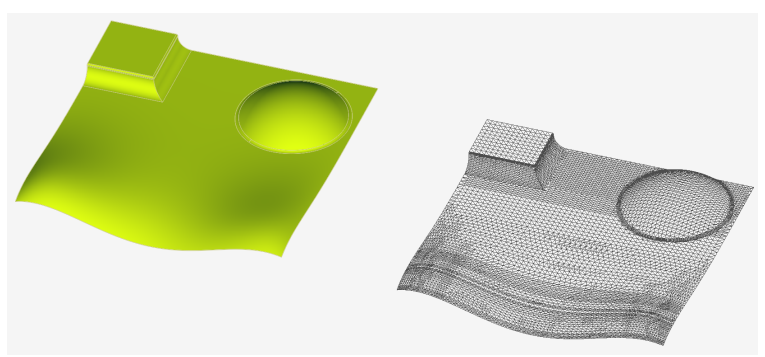

Fig. 2 Surface modeling

In this study, the proposed method for hole-filling considers the deformation of the object's CAD model to best approximate the scanned data. The CAD model is represented as a triangular mesh (STL format) (Fig. 2 ), for which $\{N\}$ is the set of nodes and $\{P\}$ the set of beams defining the triangular facets of the mesh. The mesh deformation is performed according to a physicallybased approach based on energy minimization. To preserve curvature continuity, all types of solicitations are considered here: torsion, bending and stretching.

The big picture of the method proposed to deform the mesh of the nominal part toward the measured

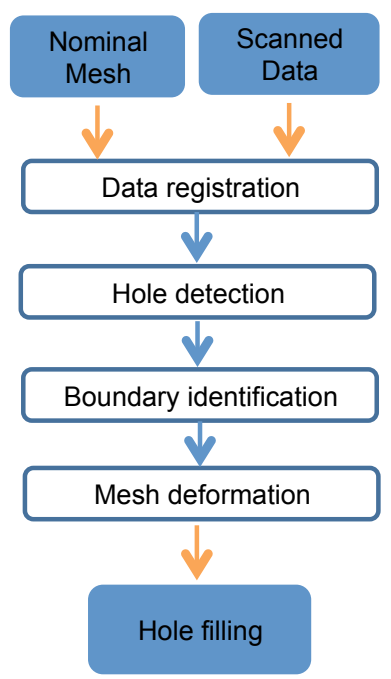

Fig. 3 Proposed approach

data is displayed in Fig.3. First, a registration step is required to globally align the mesh onto the scanned data. Indeed, to identify holes and perform a deformation that preserves the part shape, a good match between the measured and the nominal features is necessary. To make this registration, numerous methods exist [25],[22],[4], more generally based on the well-known ICP algorithm [3]. In the present study, the HT-ICP algorithm is adopted. The second and the third steps focus on hole detection and boundary identification. Indeed, boundaries limit the zones for which the deformation will be performed. The last step is the mesh deformation based on stress-strain energy minimization. The energy minimization relies on a mechanical modeling which is detailed in the next section.

\section{Mechanical modeling for energy minimization}

The CAD model is represented as a triangular mesh. Each edge defining a facet is considered as a cylindrical beam which is characterized by its length $L$ and its circular section $S$. The mechanical characteristics are defined by $(E, G, I, I o)$ respectively the Young's modulus, the torsional modulus, the second moment and the polar second moment.

\begin{tabular}{lll}
\hline & DOF & Characteristics \\
\hline Tension & $u$ & $E . S$ \\
Torsion & $\alpha$ & $G . I o$ \\
Bending in plane $(\mathrm{X}, \mathrm{Y})$ & $v$ et $\beta$ & $E . I$ \\
Bending in plane $(\mathrm{X}, \mathrm{Z})$ & $w$ et $\gamma$ & $E . I$ \\
\hline
\end{tabular}

Table 1 Degree of Freedom (DOF) description 


\subsection{Settings}

Let us consider the set of $m$ beams $\left\{P^{k}\right\}_{[1, m]}$ and the set of $n$ nodes $\left\{N_{i}\right\}_{[1, n]}$ defining beam's extremities. The displacement of each node is defined by 6 components, 3 accounting for the translation $\left(u_{i}, v_{i}, w_{i}\right)$ and 3 others for the rotation $\left(\alpha_{i}, \beta_{i}, \gamma_{i}\right)$. Data can be expressed in the global frame $\mathcal{R}$ as well as in the local frame $\mathcal{R}^{k}$ associated to the beam $k$ (Fig. 4). Hence, the following parameters can be defined:

- $U_{i}^{k}$ : Node $i$ displacement in the local frame $\mathcal{R}^{k}$.

$$
U_{i}^{k}=\left[\begin{array}{lllll}
u_{i}^{k} & v_{i}^{k} & w_{i}^{k} & \alpha_{i}^{k} \beta_{i}^{k} \gamma_{i}^{k}
\end{array}\right]_{\mathcal{R}^{k}}^{t} .
$$

- $U_{i}$ : Node $i$ displacement in the global frame $\mathcal{R}$.

- $F_{(k, i)}^{k}$ : External force applied to the beam $k$ at the node $i$ in the local frame.

- $F_{(k, i)}$ : External force applied to the beam $k$ at the node $i$ in the global frame.

Each beam $k$ is assumed to be axisymmetric and is defined by its mechanical $\left(E^{k}, G^{k}\right)$ and geometrical $\left(S_{k}, L_{k}, I_{k}, I o_{k}\right)$ characteristics. The lattice deformation is completely defined by the global force , $F=$ $\left[F_{1} \cdots F_{m}\right]^{t}$ and the node displacement $U=\left[U_{1} \cdots U_{m}\right]^{t}$ expressed in the global frame $\mathcal{R}$.

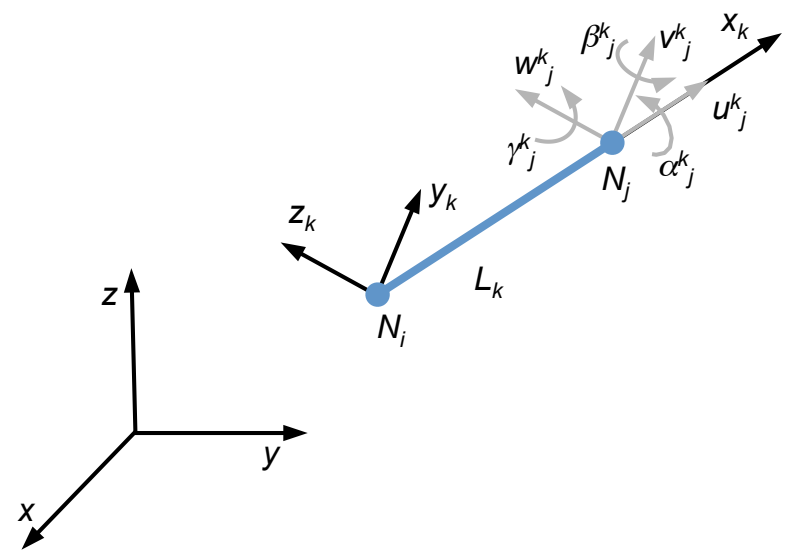

Fig. 4 Beam modeling

\subsection{Stiffness matrix definition}

For simplification reasons, we introduce the total force $\hat{F}_{k}^{k}=\left[F_{(k, i)}^{k} F_{(k, j)}^{k}\right]^{t}$ applied to the beam $k$ at each extremity and the displacement $\hat{U}_{k}^{k}=\left[U_{i}^{k} U_{j}^{k}\right]^{t}$ of each beam extremity. Hence, the local stiffness matrix of the beam $k$ is defined such as $\hat{F}_{k}^{k}=K_{k}^{k} \cdot \hat{U}_{k}^{k}$ with:

$K_{k}^{k}=\left[\begin{array}{c|c}A_{k}^{k} & C_{k}^{k} \\ \hline\left(C_{k}^{k}\right)^{t} & B_{k}^{k}\end{array}\right]$ where the components of $A_{k}^{k}, C_{k}^{k}$, and $B_{k}^{k}$ calculated by EulerBernoulli beam theory are given in appendix A. The transformation from the global frame $\mathcal{R}$ to the local frame $\mathcal{R}^{k}$ of the beam $k$ can be expressed thanks to the Euler's angles $\theta_{k}$ and $\phi_{k}$ giving the matrix $M_{\mathcal{R} \mathcal{R}^{k}}=$ $R_{y}\left(\frac{\pi}{2}-\phi_{k}\right) \cdot R_{z}\left(\theta_{k}\right)$ (Fig. 5). The external forces and the displacement can be easily expressed in the global frame by $U_{i}=M_{\mathcal{R} \mathcal{R}^{k}}^{*} \cdot U_{i}^{k}$ and $F_{(k, i)}=M_{\mathcal{R}^{k}}^{*} \cdot F_{i}^{k}$ with :

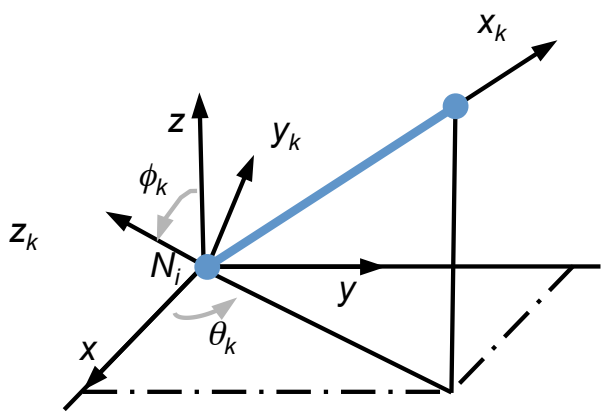

Fig. 5 Frame description

$M_{\mathcal{R}^{k}}^{*}=\left[\begin{array}{c|c}M_{\mathcal{R} \mathcal{R}^{k}} & 0 \\ \hline 0 & M_{\mathcal{R} \mathcal{R}^{k}}\end{array}\right]$

It is thus possible to define the stiffness matrix in the global frame to calculate the relationship $\hat{F}_{k}=K_{k} \cdot \hat{U}_{k}$ :

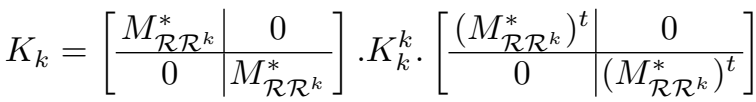

\subsection{Energy balance}

For each beam $k$, the deformation energy is defined by $E_{k}^{d e f}=\frac{1}{2} \cdot \hat{U}_{k}^{t} \cdot K_{k} \cdot \hat{U}_{k}$. Hence the total energy of deformation is defined by:

$E_{\text {tot }}^{\text {def }}=\sum_{k \in[1, n]} \frac{1}{2} \cdot \hat{U}_{k}^{t} \cdot K_{k} \cdot \hat{U}_{k}$

The global stiffness matrix $K$ is calculated by assembling all the stiffness matrices. Therefore, the total energy of deformation becomes:

$E_{\text {tot }}^{\text {def }}=\frac{1}{2} \cdot U^{t} \cdot K \cdot U$

The solution of the deformation problem consists in the minimization of the total energy. In our case, as the external forces are null the mechanical work $W^{\text {ext }}=$ $U^{t} . F$, so the solution that minimizes the strain energy is the one that minimizes the energy of deformation. The resolution of such a problem is a classic quadratic optimization problem which is solved using Matlab. 
The mesh deformation is performed by imposing displacements to the mesh nodes that do not correspond to holes and by computing the displacement of the other nodes by minimization of the total energy $E_{\text {tot }}^{\text {def }}$. Let us remember here, that each displacement includes 3 translations and 3 rotations. These 6 degrees of freedom involve both the shape preservation and the continuous evolution of the curvature. On the other hand, mesh deformation calculation can be time-consuming. Indeed, for a classical part (Fig. 2), the mesh includes about $10^{5}$ nodes each having 6 degrees of freedom. The originality of our approach is thus to reduce the minimization problem to zones of interest defined from digitized hole neighborhoods. Finding the holes is thus an essential step in the overall approach as we will see now in more details.

\section{General Approach}

The general approach consists of three main steps (figure 1.2): data registration, hole detection and boundary identification, and mesh deformation. The input data are a nominal mesh and a digitized point cloud corresponding to the scanned data. Finally, the nominal mesh is deformed to match the digitized point cloud which involves the hole filling.

\subsection{Data registration}

The first step of the approach is a global data registration between the scanned data and the nominal mesh to ensure the correspondence between scanned and nominal features. This step, necessarily performed prior to the mesh deformation enables the point cloud to be close to the nominal mesh. Global registration is achieved using a HT-ICP algorithm [4]. The result is considered satisfactory if the registration error is less than the error imputed to the manufacturing process and/or the digitizing error.

\subsection{Hole detection and boundary detection}

As point clouds obtained from scanning are generally dense and heterogeneous with a lack of continuity [14], finding digitizing holes could be a long and tedious task. To simplify this issue, we propose to take advantage of the initial data registration which leads to a good match between the scanned data and the nominal mesh. Holes are thus identified by considering points that belongs to the nominal mesh for which no equivalent exist in the point cloud.
The main hypothesis here is that the hole dimension is greater than the mesh size. Let us consider a point $N$ of the nominal mesh, and its associated normal vector (Fig. 6). The $q$ closest points to $N$ belonging to the point cloud are identified. A local mesh is built from these $q$ points, and the intersection between the line passing through $N$ and directed along the normal vector is calculated. If the intersection does not exist, as for point $N_{1}$ in figure 6 , the point $N$ is identified as a hole point, and the beams linked to this node are classified as beams to be deformed (in red in figure 6). This hole detection method turns out to be efficient and simple to implement as it is based on the calculation of the vector normal. The facets linked with the considered point are selected, and the normal vector is estimated by calculating the average of the normal vectors of the previously defined facets. Note that such a method involves the reduction of the mesh to be deformed to a restriction defined by the digitized holes.

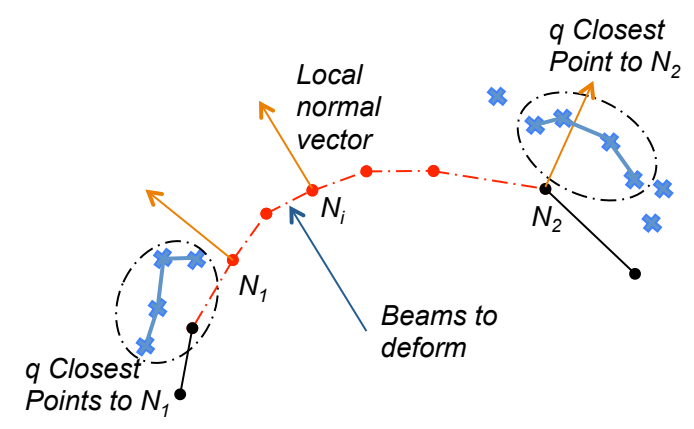

Fig. 6 Hole detection and mesh reduction

\subsection{Mesh deformation}

To preserve the curvature evolution between the deformed regions and the nominal mesh, it seems relevant to increase the region to be deformed (Fig. 7). Therefore, a ring around the border is defined. The ring's width is defined according to the mesh's size; in this study, the width is equal to three times the mesh's size. The nodes of the nominal mesh that are added to the ring necessarily have their counterpart in the scanned point cloud. For such nodes, a corresponding point can be defined in the scanned point cloud by considering the barycentre of the $q$ nearest points to the given node.

For each node belonging to the border zone (in orange in Fig. 7), the displacement $\mathbf{u}$ is imposed and must be calculated. For such nodes, the intersection between the straight line passing through the point and directed along the vector normal and the mesh created by the 


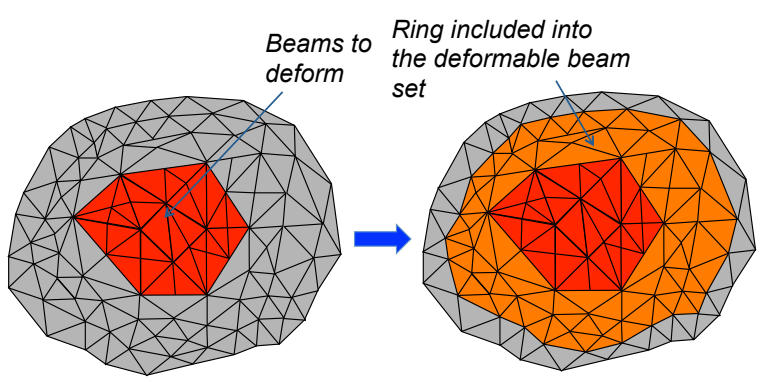

Fig. 7 Boundary definition

$q$ closest points exists (see section 3.2. The displacement is the one that makes the studied node perfectly matched with its corresponding point: $\mathbf{u}_{i}=\lambda . \mathbf{n}_{i}$ with $\mathbf{n}_{i}$ the normal vector. This defines the generalized displacement of the node $i$ in the global frame that is introduced in the energy minimization problem (eq. 6):

$U_{i}=\left[\begin{array}{lllllll}\lambda . \mathbf{n}_{i x} & \lambda . \mathbf{n}_{i y} & \lambda . \mathbf{n}_{i z} & 0 & 0 & 0\end{array}\right]_{\mathcal{R}}^{t}$.

For other points (in grey in Fig. 7), the displacement is used to reduce the gap between the nominal mesh and the measured points.

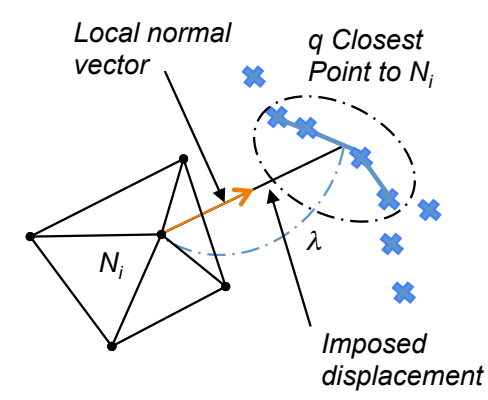

Fig. 8 Displacement computation

The minimization problem thus leads to the deformation of the nominal STL mesh to match the point cloud involving the propagation of the deformation to the digitized holes. Such an approach ensures the preservation of the general shape as the deformation is only applied to the zones of interest around the digitized holes. The management of the curvature continuity at the vicinity of the junction is done thanks to the control of the 3 rotations at each node.

\section{Application}

\subsection{Study of a sculptured part}

The method is applied to a sculptured surface made by 3D printing (ABS plastic part) (Fig. 2) bounded by a 100x100x15 $\mathrm{mm}^{3}$ box. The part is scanned using a laser scanner Kreon KZ25 mounted on a Coordinate Measuring Machine (CMM). The manufacturer announces an accuracy of $15 \mu \mathrm{m}$. Only one scanner orientation is used during the scanning process in order to reduce the digitizing noise. Under these conditions the maximal digitizing noise is estimated to be $72 \mu \mathrm{m}$ [26], [1]. After the scanning is done, artificial holes are added to the actual ones (Fig. 9). To assess our method, the holes are filled using a CAD software (CATIA C) for which the mesh size is equivalent to that used in the nominal mesh. The mesh that is filled using the aforementioned CAD software is compared to the initial point cloud (Fig. 10). As shown in the figure, the CAD software does not succeed in hole filling as some holes remain unfilled. Furthermore, deviations with the initial mesh can reach up to $1.6 \mathrm{~mm}$ which can be penalizing for some applications. Lastly, one can see that curvature continuity is not ensured and so the overall shape is not preserved. The proposed morphing method is applied. Deviations between the morphed mesh and the measured point cloud are displayed in figure 11 and a comparison between the CAD-filling approach and our approach is proposed in table 2 . Since the distance between the scanned data and the CAD-filled mesh are identical outside the hole areas, the mean distance and the standard deviation are closed to those obtained with the morphing-based approach. However, the maximal distance is strongly reduced with our algorithm (three times lesser). Furthermore, figure 11 clearly highlights smoothing and overall shape preservation.

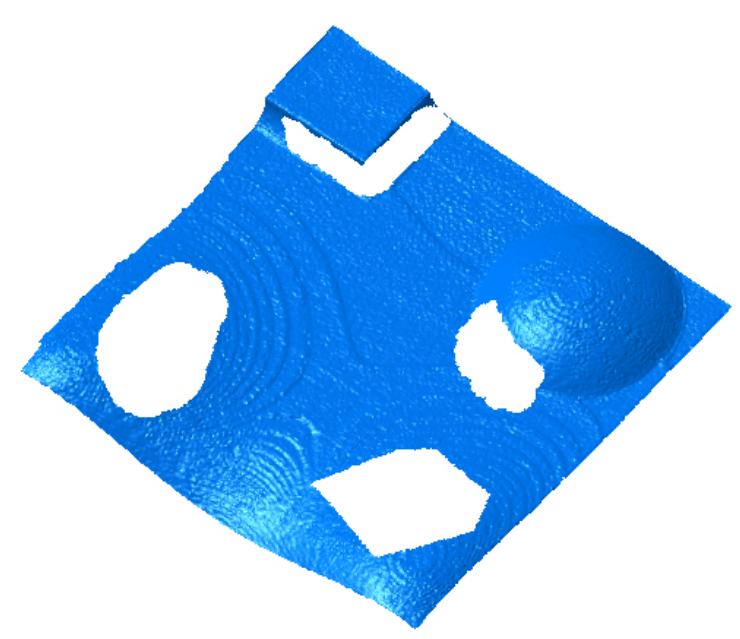

Fig. 9 Digitized point cloud with artificial holes

Nevertheless, high deviations can be observed on the edges of the part. This is likely due to the normal vector calculation, which is deficient when it comes to mesh edges for which there is a lack of information. 

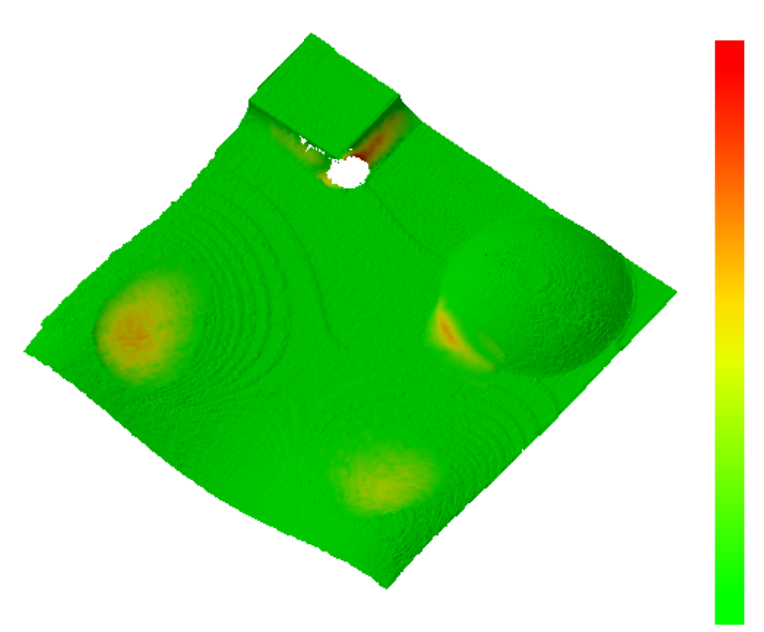

$1,6 \mathrm{~mm}$

$1,4 \mathrm{~m} \mathrm{~m}$

$1,3 \mathrm{~mm}$

$1,1 \mathrm{~mm}$

$0,96 \mathrm{~m} \mathrm{~m}$

$0,8 \mathrm{~mm}$

$0,64 \mathrm{~mm}$

$0,48 \mathrm{~m} \mathrm{~m}$

$0,48 \mathrm{~mm}$

$0,32 \mathrm{~mm}$

$0,16 \mathrm{~mm}$

$0 \mathrm{~mm}$

Fig. 10 Deviations between the CAD-filled mesh and the digitized point cloud

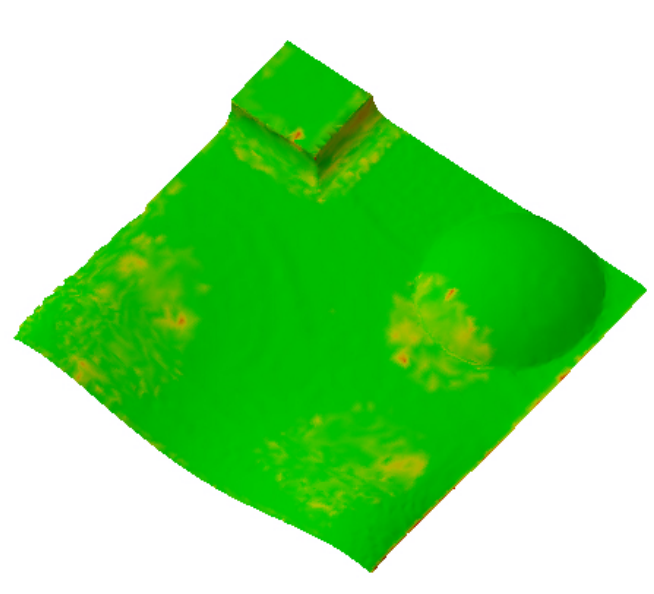

$0,55 \mathrm{~mm}$

$0,49 \mathrm{~mm}$

$0,44 \mathrm{~mm}$

$0,38 \mathrm{~mm}$

$0,33 \mathrm{~mm}$

$0,27 \mathrm{~mm}$

$0,22 \mathrm{~mm}$

$0,16 \mathrm{~mm}$

$0,11 \mathrm{~mm}$

$0,055 \mathrm{~mm}$

$0 \mathrm{~mm}$

Fig. 11 Deviations between the morphed mesh and the digitized point cloud

\begin{tabular}{lcc}
\hline & Cad Filled & Morphing \\
\hline Maximal deviation & $1.6 \mathrm{~mm}$ & $0.55 \mathrm{~mm}$ \\
Mean Deviation & $0.0085 \mathrm{~mm}$ & $0.044 \mathrm{~mm}$ \\
Standard Deviation & $0.067 \mathrm{~mm}$ & $0.073 \mathrm{~mm}$ \\
\hline
\end{tabular}

Table 2 Results Analysis

Let us see now, the interest of our algorithm for point exploitation, in the particular case of inertia parameter calculation for crankshaft balancing.

\subsection{Improving crankshaft balancing}

This illustration is directly linked with a new method for crankshaft balancing based on non-contact measurement. In this method, once the crankshaft is digitized using a laser-plane scanner a STL mesh is directly built from the digitized points. This mesh, which must define a closed structure, is the support for a voxelisation algorithm [16]. The voxelisation algorithm delivers a 3D structure of voxels, each one being assigned with an elementary mass. The calculation of inertia parameters is carried out considering this $3 \mathrm{D}$ structure made of concentrated masses located at the center of each voxel. This method has proven to be efficient for the estimation of the specific unbalances if the digitized point cloud is a complete representation of the shape [26]. Digitized holes can alter unbalance calculation. Indeed, crankshafts are very complex parts, which generally require numerous sensor orientations to access all the surfaces to be measured. As this process is timeconsuming, the idea is to limit the number of sensor orientations, and to fill the holes with the morphingbased approach we propose. '

\begin{tabular}{ll}
\hline Number of nodes of initial mesh & 12718 \\
Number of nodes of restricted mesh & 2775 \\
Number of nodes of the scanned mesh & 545007 \\
\hline
\end{tabular}

Table 3 Computation parameters

For this purpose, the digitizing of the crankshaft is simulated from the CAD model. First, the defects of the manufacturing process are simulated so that the crankshaft has unbalance defects. Rough crankshafts are conventionally obtained by forging. Associated defects are simulated by performing an expansion of $3 \%$ of the nominal CAD model and by carrying out a $0.5 \mathrm{~mm}$ shift between the two sections located on both sides of the symmetry plane. To simplify the representation, only a $100 \mathrm{~mm}$ portion of the crankshaft is studied (Fig. 12(a)). Mesh specifications are detailed in table 3 . These defects generate unbalances that are calculated in the two reference planes located on either extremity (Tab. 4).

Once the defects are simulated, the mesh is calculated from the modified CAD model. To simulate digitizing holes, holes are added to the mesh (represented in purple in Fig. 12(a)) corresponding to areas that are difficult to scan [26]. Our algorithm is thus applied. Results highlight a mean deviation of $8.7 \mu \mathrm{m}$ and a standard deviation of $92 \mu \mathrm{m}$ (Fig. 13). Unbalances are calculated for the morphed mesh. As displayed in table 4, the values calculated after morphing are close to the actual values. Indeed, the estimated unbalances are determined with an error of less than $1.5 \%$. To enhance the efficiency of our approach, unbalances obtained using a classical CAD-filling are also calculated and compared to the actual ones. In this case, the error is up 
to $3 \%$ which could be penalizing for the further balancing operation. Therefore, with the morphed-based approach to fill digitized holes, it is possible to preserve the overall shape of the digitized object, and then, it is also possible to conduct inertia calculations with an error of less than $1.5 \%$ relatively to the actual values.

\begin{tabular}{lll}
\hline Part & Unbalance 1 (g.cm) & Unbalance 2 (g.cm) \\
\hline Nominal mesh & 261.7 & 261.5 \\
Morphing & 258.9 & 258.3 \\
Cad filled & 253.9 & 253.8 \\
\hline
\end{tabular}

Table 4 Unbalance in reference planes

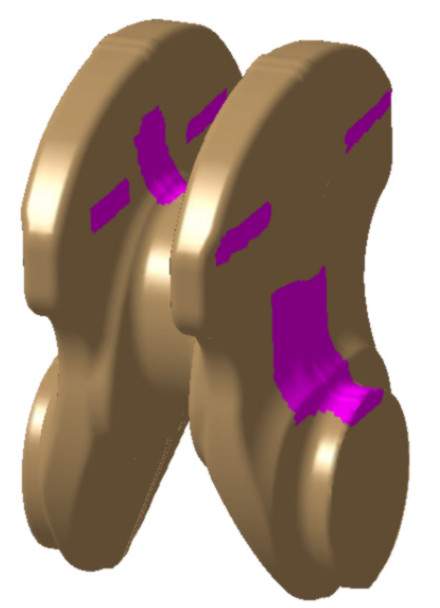

(a) Representation of the nominal mesh

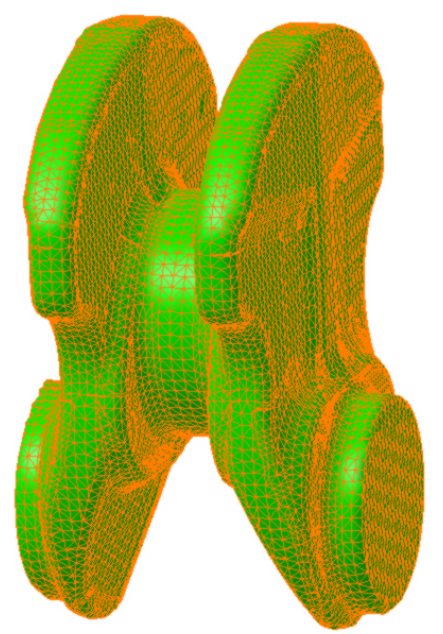

(b) Representation of the morphed mesh

Fig. 12 Representation of the studied part

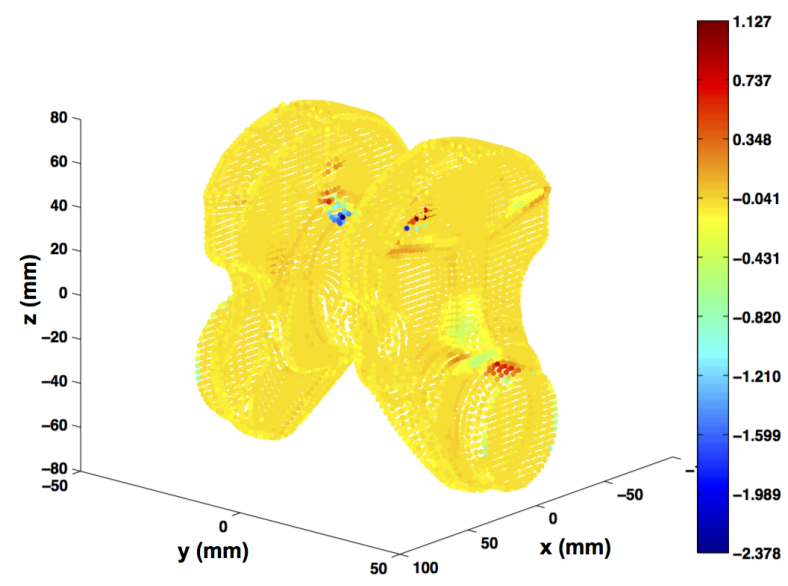

Fig. 13 Deviation (mm) between nominal and morphed crankshaft

\subsection{Application to digitized data}

The method is applied to actual digitized data. The crankshaft is digitized using a structured light sensor (Gom Atos III) positioned thanks to a tripod.Under these conditions, the maximal digitizing noise is estimated at $18 \mu \mathrm{m}$ [26]. From previous work [11], we know that fifty viewpoints are necessary to reach completeness. However, digitizing holes are present in the point cloud in non-accessible areas. To clarify the representation, only the arm connecting the crankpin to the crank journal and one of the cylinders machined at both extremities are specifically studied (Fig. 15(b) and 16(b)).

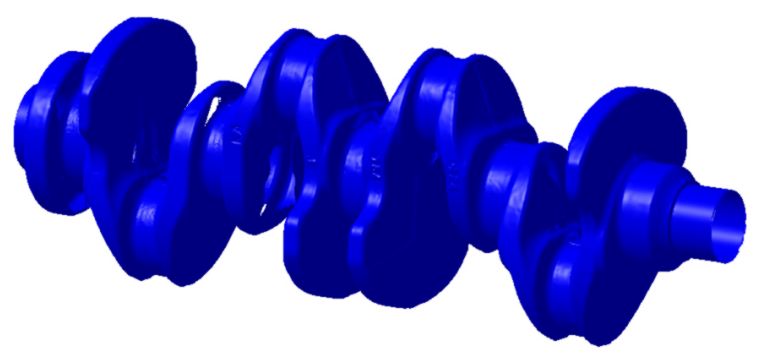

Fig. 14 Digitized crankshaft

The arm is bounded by a $85 \times 40 \times 110 \mathrm{~mm}^{3}$ box. According to the proposed method, the nominal mesh (Fig. 15(a)) is deformed, and the morphed mesh is displayed in Fig. 15(c) and 15(d). Point identified as holes (section 3.2)) are represented in red, and points of the border used to impose displacements (section 3.3) are represented in orange. Despite the complexity of the hole boundary (Fig. 15(b)) and of the digitizing noise, the mesh morphing leads to a satisfactory result as the shape is clearly preserved, along with the curvature evo- 
lution $15(\mathrm{~d})$. The morphed mesh can replace the point cloud for further point exploitation. This result highlights the performance of the method for filling actual digitized holes.

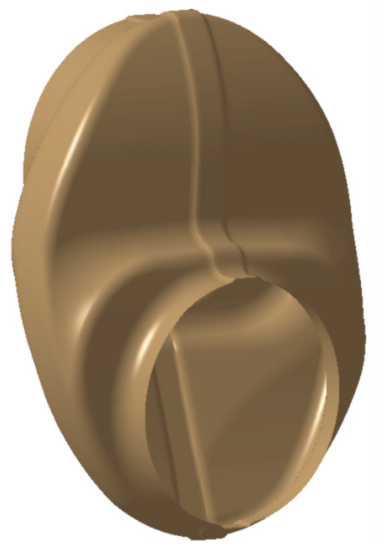

(a) Nominal mesh

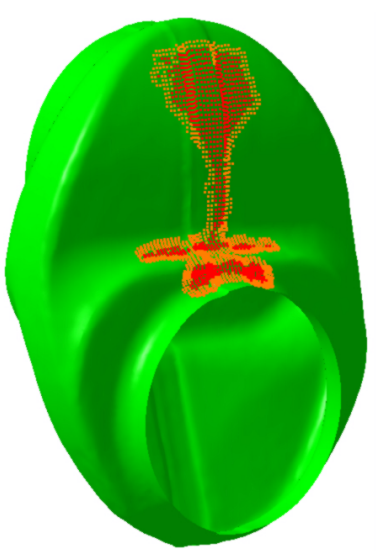

(c) Morphed mesh

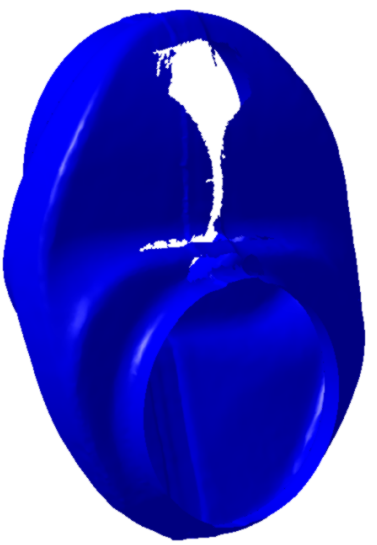

(b) Digitized mesh

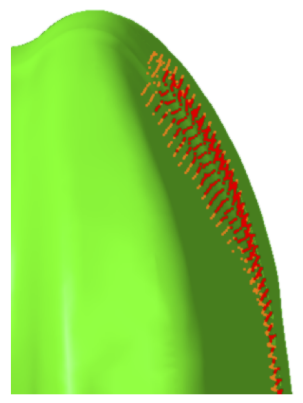

(d) Detail of hole filling
Fig. 15 Representation of studied part of the crankshaft

Regarding the second part, it consists of a $41 \mathrm{~mm}$ diameter and $19 \mathrm{~mm}$ length cylinder (Fig 16(a)). In this case, the lack of data corresponds to the top plane (Fig 16(b)). As displayed in the figure, only a few points are digitized on the plane surface, which involves that only a few points can be used to define the border of the planar portion. In addition, due to the tangency discontinuity between the plane and the cylinder, digitizing noise is high in this area. As a result, the calculation of the imposed displacements is heavily altered, and the resulting morphed mesh is incorrect (Fig 16(c)).

\subsection{Discussion}

The applications we proposed highlight the relevance of the morphing-based approach based on the CAD model (a) Nominal mesh

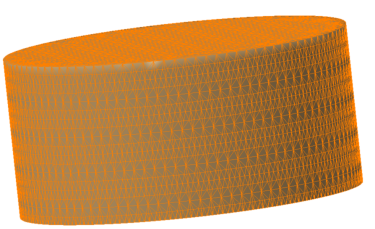

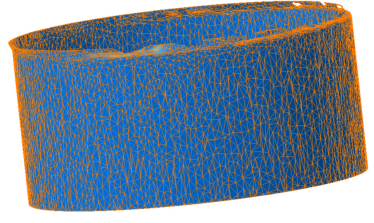

(b) Digitized points

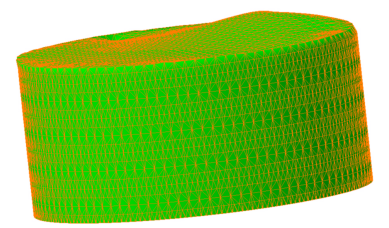

(c) Morphed mesh

Fig. 16 The studied cylinder

to fill digitizing holes prior to point exploitation. This is clearly illustrated through the crankshaft balancing example. When digitized data are used, although digitized holes present a complex geometry, and despite digitizing noise, the morphed mesh can be calculated. The general shape is preserved and all the holes are filled considering curvature continuity at the junctions. However, the method present some limits. First, the manufacturing process is assumed to be controlled, i.e. the shape of the actual part is not very different from the shape of the nominal part. This is generally the case for machining or additive manufacturing operations as proposed here. In addition, the hole must not cover a complete feature, as it is the case with the top plane of the cylinder. The great lack of points on the surface involves a bad shape prediction. Finally, digitizing noise can be penalizing at the vicinity of the part edges for which the evaluation of the normal vector can be deficient.

\section{Conclusion}

A complete approach has been presented in this paper for filling holes in point clouds with a priori knowledge of the CAD model. This approach relies on the morphing of the CAD model represented as a mesh according to the minimization of the mechanical energy. The mesh is associated to a beam lattice and, to preserve curvature continuity, all types of solicitations are considered (torsion, bending and stretching). Hole-filling with shape preservation is particularly essential to facilitate applications such as Rapid Prototyping, Finite Element Analysis, or inertia calculations as proposed in this paper. To prevent substantial computational time, a method of restriction of the meshes to the digitized hole areas has been developed. This method preserves 
the curvature connection between the deformed areas and the nominal mesh. As crankshaft balancing using optical means is nowadays a challenging issue, the application of the proposed approach concerns the calculation of unbalances using incomplete simulated digitized data. Using our approach, in comparison with classical CAD-filling methods, showed a significant decrease in unbalance determination. The relevance of the morphing-based approach based on the CAD model to fill digitizing holes is also illustrated by actual digitized data. The method is efficient when the manufacturing process is controlled and when data are not affected by the digitizing noise (when it remains inferior to the mean value of the sensor noise. The morphed mesh can thus replace the point cloud for further point exploitation.Such a method could also help in the definition of digitizing strategy for complex parts. Indeed, complete digitizing is not necessary anymore when digitizing holes can be accurately filled with shape preservation.

\section{References}

1. Audfray, N., Mehdi-souzani, C., Lartigue, C.: A novel approach for $3 \mathrm{~d}$ part inspection using laser-plane sensors. 12th CIRP Conference on Computer Aided Tolerancing pp. 1-3 (2012)

2. Bernard, A.: Virtual engineering: Methods and tools. Proceedings of the Institution of Mechanical Engineers, Part B: Journal of Engineering Manufacture 219, 413$421(2005)$

3. Besl, P.J., Kay., N.D.M.: A method for registration of 3d shape. IEEE Transactions on Pattern Analysis and Machine Intelligence 14(5) (1992)

4. Chen, J., Wu, X., Wang, M.Y., Li, X.: 3d shape modeling using a self-developed hand-held $3 \mathrm{~d}$ laser scanner and an efficient ht-icp point cloud registration algorithm. Optics \& Laser Technology 45, 414-423 (2013)

5. Choi, J., Szymczak, A.: Fitting solid meshes to animated surfaces using linear elasticity. ACM Transactions on Graphics - TOG 28(1), 1-10 (2009)

6. Derigent, W., Chapotot, E., Ris, G., S.Remy, Bernard, A.: 3 d digitizing strategy planning approach based on a cad model. Journal of Computing and Information Science in Engineering 7(1), 10-19 (2007)

7. Galantucci, L.M., Percoco, G., Spina, R.: Evaluation of rapid prototypes obtained from reverse engineering. Proceedings of the Institution of Mechanical Engineers, Part B: Journal of Engineering Manufacture 217, 1543-1552 (2003)

8. Grassia, L., Hraiechb, N., Schileoa, E., Ansalonia, M., M.Rochetteb, Vicecontia, M.: Evaluation of the generality and accuracy of a new mesh morphing procedure for the human femur. Medical Engineering and Physics 33, $112-120(2011)$

9. Jun, Y.: A piecewise hole filling algorithm in reverse engineering. Computer-Aided Design 37(2), 263-270 (2005)

10. Kho, Y., Garland, M.: Sketching mesh deformations. Proceedings of the ACM Symposium on Interactive 3D Graphics (2005)
11. Lartigue, C., Quinsat, Y., Mehdi-souzani, C., ZuqueteGuarato, A., , Tabibian, S.: Voxel-based path planning for 3d scanning of mechanical parts. Computer-Aided Design and Applications 11(3), 220-227 (2014)

12. Li, Z., Meek, D.S., Wlaton, D.: Polynomial blending in a mesh hole-filling application. Computer-Aided Design 42(4), 340-349 (2010)

13. Martins, F.A.R., Garci-Bermejo, J., Casanova, E.Z., Gonzalez, J.R.P.: Automated 3d surface scanning based on cad model,mechatronics. Mechatronics 15, 837-857 (2005)

14. Medhi-Souzani, C., Thiebaut, F., Lartigue, C.: Scan planning strategy for a general digitized surface. Journal of Computing and Information Science in Engineering 6(4), 331-340 (2006)

15. Panchetti, M., Pernot, J.P., Véron, P.: Towards recovery of complex shapes in meshes using digital images for reverse engineering applications. Computer-Aided Design 42(8), 693-707 (2010)

16. Patil, S., Ravi., B.: Voxel-based representation, display and thickness analysis of intricate shapes. Ninth International Conference on Computer Aided Design and Computer Graphics (2005)

17. Pernot, J.P., Moraru, G., Vron, P.: Filling holes in meshes using a mechanical model to simulate the curvature variation minimization. Computers and Graphics 30(6), 892$902(2006)$

18. Soren, L., Kjellander, J.: Path planning for laser scanning with an industrial robot. Robotics and Autonomous Systems 56(7), 615-624 (2008)

19. Wang, J., Oliveira, M.: Filling holes on locally smooth surfaces reconstructed from point clouds. Image and Vision Computing 25(1), 103-113 (2007)

20. Wang, L.C., Y.-C., H.: Hole filling of triangular mesh segments using systematic grey prediction. ComputerAided Design 44(12), 1182-1189 (2012)

21. Wang, X., Liu, X., L.Lu, Cao, B.L.J., Yinf, B., Shig, X.: Automatic hole-filling of cad models with feature preserving, computers and graphics. Computers and Graphics 36(2), 101-110 (2012)

22. Wu, X., Chen, J., Wang, M.Y., Li, X.: Hong-tan based icp registration for partially overlapping range images. VRCAI (2011)

23. Zheng, S.X., Li, J., Sun, Q.F.: A novel 3d morphing approach for tooth occlusal surface reconstruction. Computer-Aided Design 43(3), 293-302 (2011)

24. Zhou, X., Su, Z., Liu, X.: A novel energy-based method for mesh deformation. International Conference on Educational and Information Technology pp. 153-156 (2010)

25. Zhu, L., Barhak, J., Srivatsan, V., Katzr, R.: Efficient registration for precision inspection of free-form surfaces. Int J Adv Manuf Technol 32, 505-515 (2007)

26. Zuquete-Guarato, A., Mehdi-souzani, C., Quinsat, Y., Lartigue, C., Sabri, L.: Towards a new concept of in-line crankshaft balancing by contact less measurement: process for selecting the best digitizing system. 11th Biennial Conference on Engineering Systems and Design Analysis (2012)

\section{Appendix A: stiffness matrix description}

$K_{k}^{k}=\left[\begin{array}{c|c}A_{k}^{k} & C_{k}^{k} \\ \hline\left(C_{k}^{k}\right)^{t} & B_{k}^{k}\end{array}\right]$ 


$$
\begin{aligned}
A_{k}^{k}= & {\left[\begin{array}{cccccc}
\frac{E^{k} \cdot S^{k}}{L^{k}} & 0 & 0 & 0 & 0 & 0 \\
0 & \frac{12 \cdot E^{k} \cdot I^{k}}{\left(L^{k}\right)^{3}} & 0 & 0 & 0 & \frac{6 \cdot E^{k} \cdot I^{k}}{\left(L^{k}\right)^{2}} \\
0 & 0 & \frac{12 \cdot E^{k} \cdot I^{k}}{\left(L^{k}\right)^{3}} & 0 & -\frac{6 \cdot E^{k} \cdot I^{k}}{\left(L^{k}\right)^{2}} & 0 \\
0 & 0 & 0 & \frac{G^{k} \cdot I O^{k}}{L^{k}} & 0 & 0 \\
0 & 0 & -\frac{6 \cdot E^{k} \cdot I^{k}}{\left(L^{k}\right)^{2}} & 0 & \frac{4 \cdot E^{k} \cdot I^{k}}{L^{k}} & 0 \\
0 & \frac{6 \cdot E^{k} \cdot I^{k}}{\left(L^{k}\right)^{2}} & 0 & 0 & 0 & \frac{4 \cdot E^{k} \cdot I^{k}}{L^{k}}
\end{array}\right](9) } \\
B_{k}^{k}= & {\left[\begin{array}{cccccc}
\frac{E^{k} \cdot S^{k}}{L^{k}} & 0 & 0 & 0 & 0 & 0 \\
0 & \frac{12 \cdot E^{k} \cdot I^{k}}{\left(L^{k}\right)^{3}} & 0 & 0 & 0 & -\frac{6 \cdot E^{k} \cdot I^{k}}{\left(L^{k}\right)^{2}} \\
0 & 0 & \frac{12 \cdot E^{k} \cdot I^{k}}{\left(L^{k}\right)^{3}} & 0 & \frac{6 \cdot E^{k} \cdot I^{k}}{\left(L^{k}\right)^{2}} & 0 \\
0 & 0 & 0 & \frac{G^{k} \cdot I o^{k}}{L^{k}} & 0 & 0 \\
0 & 0 & \frac{6 \cdot E^{k} \cdot I^{k}}{\left(L^{k}\right)^{2}} & 0 & \frac{4 \cdot E^{k} \cdot I^{k}}{L^{k}} & 0 \\
0 & -\frac{6 \cdot E^{k} \cdot I^{k}}{\left(L^{k}\right)^{2}} & 0 & 0 & 0 & \frac{4 \cdot E^{k} \cdot I^{k}}{L^{k}}
\end{array}\right](10) } \\
C_{k}^{k}= & {\left[\begin{array}{cccccc}
-\frac{E^{k} \cdot S^{k}}{L^{k}} \\
0 & -\frac{12 \cdot E^{k} \cdot I^{k}}{\left(L^{k}\right)^{3}} & 0 & 0 & 0 & 0 \\
0 & 0 & -\frac{12 \cdot E^{k} \cdot I^{k}}{\left(L^{k}\right)^{3}} & 0 & 0 & \frac{6 \cdot E^{k} \cdot I^{k}}{\left(L^{k}\right)^{2}} \\
0 & 0 & 0 & -\frac{6 \cdot E^{k} \cdot I^{k} \cdot I o^{k}}{\left(L^{k}\right)^{2}} & 0 \\
0 & 0 & \frac{6 \cdot E^{k} \cdot I^{k}}{\left(L^{k}\right)^{2}} & 0 & \frac{2 \cdot E^{k} \cdot I^{k}}{L^{k}} & 0 \\
0 & -\frac{6 \cdot E^{k} \cdot I^{k}}{\left(L^{k}\right)^{2}} & 0 & 0 & 0 & \frac{2 \cdot E^{k} \cdot I^{k}}{L^{k}}
\end{array}\right](11) }
\end{aligned}
$$

\section{Appendix B: Frame transformation description}

$$
M_{\mathcal{R}^{k}}=\left[\begin{array}{cll}
\cos \left(\frac{\pi}{2}-\phi_{k}\right) & 0 & \sin \left(\frac{\pi}{2}-\phi_{k}\right) \\
0 & 1 & 0 \\
-\sin \left(\frac{\pi}{2}-\phi_{k}\right) & 0 & \cos \left(\frac{\pi}{2}-\phi_{k}\right)
\end{array}\right] \cdot\left[\begin{array}{ccc}
\cos (\alpha) & -\sin \left(\theta_{k}\right) & 0 \\
\sin \left(\theta_{k}\right) & \cos \left(\theta_{k}\right) & 0 \\
0 & 0 & 1
\end{array}\right] \text { (12) }
$$

